\title{
COLOR CONVERTING OF ENDOSCOPIC IMAGES USING DECOMPOSITION THEORY AND PRINCIPAL COMPONENT ANALYSIS
}

\author{
Keivan Ansari ${ }^{1,2}$, Alexandre Krebs ${ }^{1}$, Yannick Benezeth ${ }^{1}$ and Franck \\ Marzani ${ }^{1}$ \\ ${ }^{1}$ ImViA-Imaging and ArtificialVision,Université de Bourgogne, Dijon, \\ France \\ ${ }^{2}$ Dept. Color Imaging and Color Image Processing, Institute for Color \\ Science and Technology, Tehran, Iran
}

\begin{abstract}
Endoscopic color imaging technology has been a great improvement to assist clinicians in making better decisions since the initial introduction. In this study, a novel combined method, including quadratic objective functions for the dichromatic model by Krebs et al. and Wyszecki's spectral decomposition theory and the well-known principal component analysis technique is employed. New algorithm method working for color space converting of a conventional endoscopic color image, as a target image, with a Narrow Band Image (NBI), as a source image.

The images of the target and the source are captured under known illuminant/sensor/filters combinations, and matrix $Q$ of the decomposition theory is computed for such combinations. The intrinsic images which are extracted from the Krebs technique are multiplied by the matrix $Q$ to obtain their corresponding fundamental stimuli. Subsequently, the principal component analysis technique was applied to the obtained fundamental stimuli in order to prepare the eigenvectors of the target and the source. Finally, the first three eigenvectors of each matrix were then considered as the converting mapping matrix. The results precisely seem that the color gamut of the converted target image gets closer to the NBI image color gamut.
\end{abstract}

\section{KEYWORDS}

Color Converting, Endoscopic Imaging, Dichromatic Model, Principal Component Analysis, Decomposition Theory.

\section{INTRODUCTION}

Since the time that endoscopic imaging technology of human internal organs was introduced, the researchers of color image processing have been looking for creating methods to improve medical diagnosis[19,20]. The new methods were expected to improve the visualization of lesions and problems of the tissue under visual assessment. For instance, endoscopic images that are provided under narrow-band light sources i.e., Narrow-Band Imaging (NBI), have been clinically proved to have a higher quality of medical diagnosis in spite of the fact that they do not show the colors similar to the conditions in which the tissue is imaged with white light [1,2]. Consequently, from this point of view, correct transfer of the tissue color during digital representation of the image does not necessarily have priority anymore, but instead as

Natarajan Meghanathan et al. (Eds) : ICCSEA, WiMoA, SCAI, SPPR, InWeS, NECO - 2019 pp. 151-159, 2019. ㅇ CS \& IT-CSCP 2019

DOI: $10.5121 /$ csit.2019.91812 
our purpose, we can devise methods to change the color space of the image to increase the quality in the visual assessment of endoscopic images.

The topic of the color change of an image is a valuable one that has useful applications in color image enhancement, color constancy, color gamut mapping, and color reproduction in space telescope images, artistic images, industrial images, etc. [3-6].

One of the techniques for color changing of an image is the ability to transfer the color space of a source image to a target image. There are various and interesting researches conducted in this area as well.

Saito et al. proposed a histogram matching method between two color gamuts, based on matching the minimum and maximum values of the source image to the corresponding values of the target image [7,8]. Likewise, Gorji Kandi et al. suggested a histogram rescaling between two color gamuts using Rosenfeld-Kak histogram matching based on matching the $\mathrm{R}, \mathrm{G}$, and $\mathrm{B}$ channels of the source image to the corresponding histograms of the target image [9]. Reinhard et al. attempted to match the means and variances between the target and the source image by converting RGB into L $\alpha \beta$ color space which is developed with Ruderman et al. Afterwards, they determined the means and standard deviations for any axis separately in L $\alpha \beta$ space and eventually converted the enhanced result back to RGB [10]. Chang et al. introduced a color category-based method by classifying each pixel as one of the basic categories. Then a convex hull was produced in L $\alpha \beta$ color space for each category of the pixel sets, and the color transformation was applied with each pair of the convex hull of the same category [11]. Paris et al. proposed the Local Laplacian Pyramid method to profit the edge-preserving decomposition for the desired level of detail manipulation [12].

Abadpour et al., who used the fuzzy principal component analysis-based clustering technique (FPCAC), developed an independent color space to minimize color correlation by finding two sets of membership maps as a result of the clustering concerning the cluster parameters [13]. By contrast, a survey review by Dhanve et al. shows that the majority of methods would be producing insufficient similar color distortion and grain effect [14].

This study proposes a new method of converting color space from aNBI image to a standard endoscopic image of internal organ. The purpose is to enhance such endoscopic images to provides a better visual assessment quality for the medical diagnoses.

\section{METHOD AND RESULTS}

\subsection{Applying Dichromatic Model}

The color appearance of human internal tissues is a consequence of color, gloss, and texture. In our approach, it is required to separate the shading factor and specularity from intrinsic image for each pixel. Among various techniques, we selected and applied the Krebs et al. technique based on the quadratic objective functions to estimate dichromatic model parameters from a single color image [15]. The dichromatic model was introduced by Shafer et al. in 1985[16]. This model represents an image as a linear combination of the photometric invariants:

$$
I(u ; n)=L(\lambda)(g(u) S(u ; n)+k(u))
$$

Where in equation (1), $I$ is the input image, $\mathrm{L}$ is the illuminant multiplied by the sensor and filters sensitivity, $\mathrm{g}$ is the shading factor, $\mathrm{k}$ is the specularity, and $\mathrm{S}$ is the intrinsic image. Also, $u$ and $\lambda$ denoted the pixel location and the number of channels, respectively. Figure 1 
and Figure 2 show the dichromatic estimated $\mathrm{S}, \mathrm{g}$, and $\mathrm{k}$ of the source and target images. The source and the target are a typical example of NBI and conventional endoscopic images.

\subsection{Applying Matrix $Q$}

We assumed the source and the target images are captured under known illuminant/sensor/filters combinations, and then matrix $\mathrm{Q}$ of the decomposition theory is computed for such combination. Decomposition theory by Cohen and Kappauf was introduced and employed as an orthogonal projector, and it was attained from the Wyszecki`s spectral decomposition theory. Based on the Wyszecki hypothesis, In a series of articles, Cohen and Kappauf formalized a mathematical technique for decomposing the intrinsic image $\mathrm{S}(\mathrm{u} ; \mathrm{n})$ as a stimuli, into its fundamental stimuli, $\mathrm{S}_{\mathrm{FCS}}$ through a framework of orthogonal projectors using linear algebra[17]. Fundamental stimuli is intrinsically associated with the color values shared to all possible metamers ever to be encountered [21].
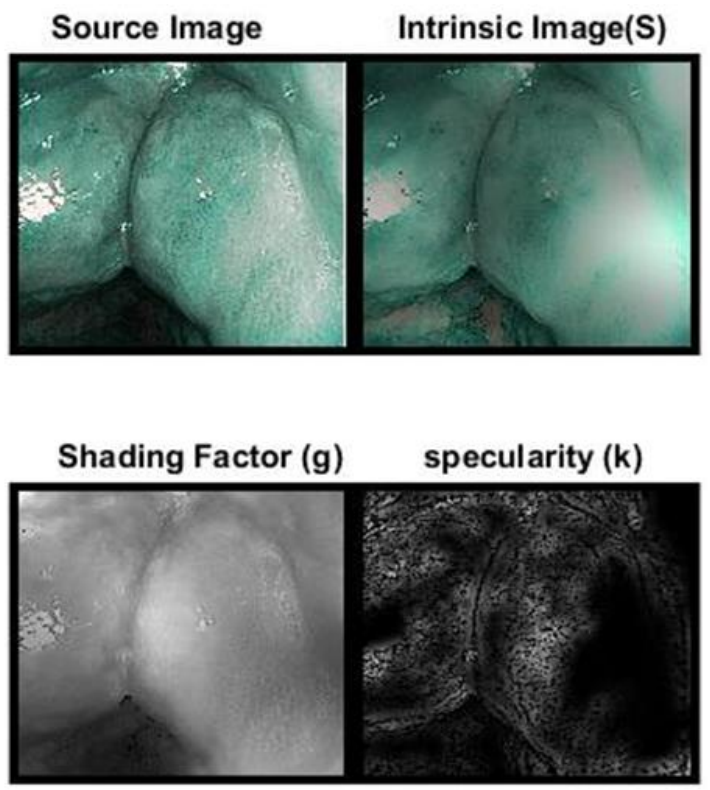

Figure 1. The Source image[2] and its intrinsic image (S), shading factor(g), and secularity $(\mathrm{k})$ using the dichromatic model estimation. 

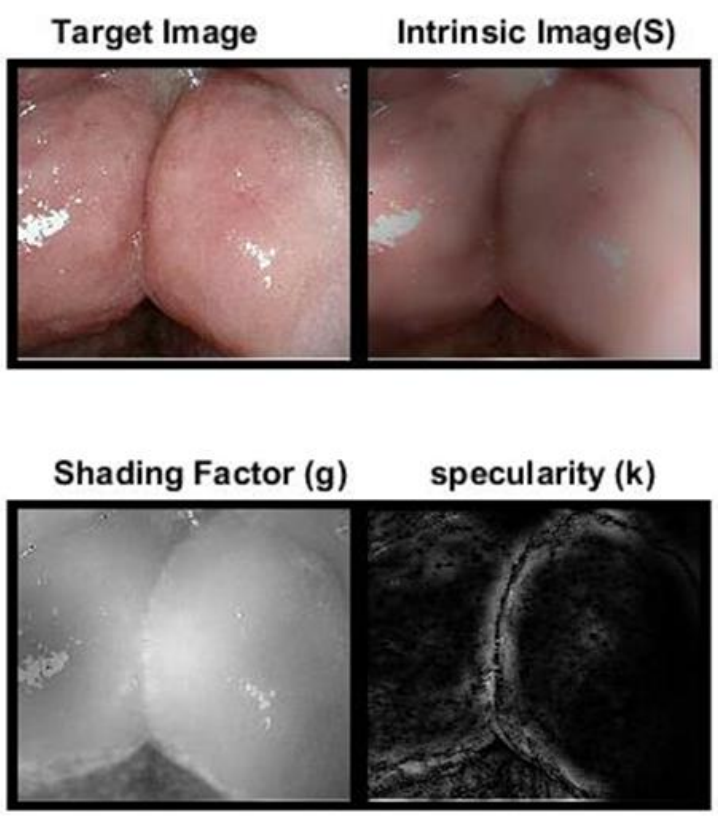

Figure 2. The Target image[2] and its intrinsic image (S), shading factor $(\mathrm{g})$, and specularity(k) using the dichromatic model estimation.

$$
S=L^{\prime} N \quad \text { (2) }
$$

By uniformly sampling for each pixel in equation (2), $\mathrm{L}$ is a $\mathrm{p} \times 3$ matrix, provides a mapping from usually large p-dimensional intervals of spectral reflectance, $\mathrm{N}$ into only threedimensional stimuli, S. Because of this tense dimensionality reduction, there are theoretically exist many different spectral reflectance $\mathrm{Ni}(\mathrm{i}=1, \ldots, \mathrm{m})$ which all can represent the same stimuli, S.

$$
L^{\prime} N_{i}=S
$$

Premultiplying any $3 \times 1$ dimensional vectors of $S$ by the $p \times 3$ matrix, $L\left(L^{\prime} L\right)^{-1}$ produce a $p \times 1$ dimensional vector of SFCS [18].

$$
L\left(L^{\prime} L\right)^{-1} L^{\prime} N_{i}=L\left(L^{\prime} L\right)^{-1} S=S_{F C S}
$$

The equality $L\left(L^{\prime} L\right)^{-1} S=$ SFCS lets compute the fundamental stimuli, SFCS from stimuli, $S$ for each pixels. It would be called matrix $\mathrm{Q}$.

$$
\text { Matrix } Q=L\left(L^{\prime} L\right)^{-1}
$$

Figure 3shows the fundamental stimuli, SFCS for each pixels of the source and the target images by computing of the corresponding $\mathrm{L}$ for illuminant/sensor/filters combinations which are employed to calculatetheirown matrixQ. 

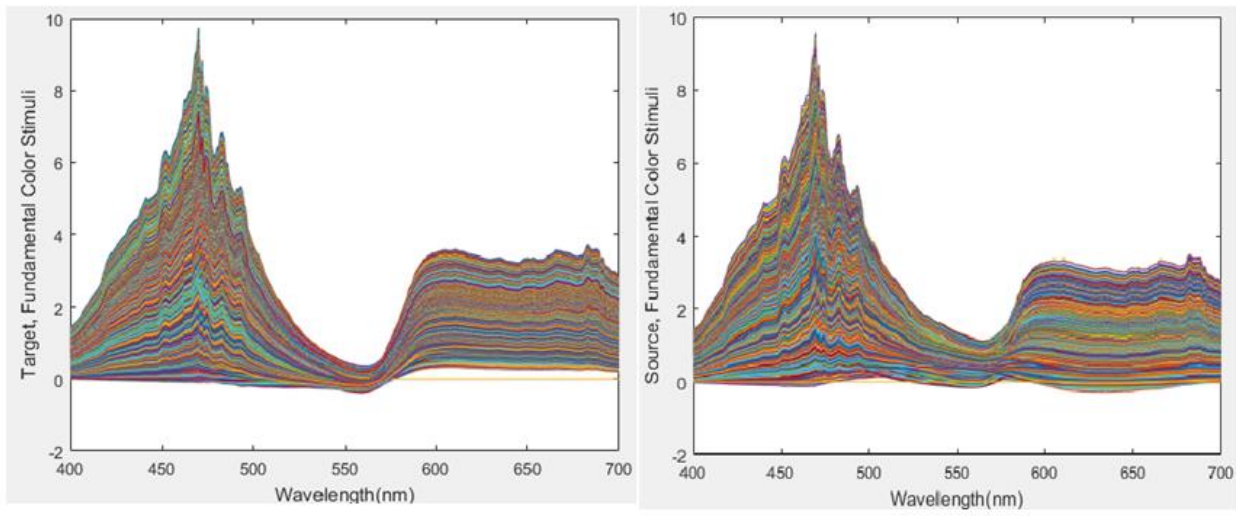

Figure 3. The fundamental stimuli $\left(\mathrm{S}_{\mathrm{FCS}}\right)$ of pixels: the source image(left), the target image(right).

\subsection{Applying Principal Component Analysis}

Then, we extructed eigenvectors and eigenvalues of the $\mathrm{S}_{\mathrm{FCS}}$ matrix of the source and the target by the well-known principal component analysis, PCA technique. Extracted eigenvectors sorted according to their corresponding eigenvalue importance. Computing cumulative index of diagonal eigenvalue matrix of the source and the target shows the first three eigenvectors, prepares enough information to fully recover the co-variance matrix of the source and the target's $S_{F C S}$. Then we compute the specifically weighted coefficient $(\alpha)$ of the PCA technique that needs to recover each $\mathrm{S}_{\mathrm{FCS}}$ belonging to the source and the target.

Consequently, color converting would be prepared by estimating new $\hat{\mathrm{S}}_{\mathrm{FCS}}$ values of the target. $\hat{\mathrm{S}}_{\mathrm{FCS}}$ of the target is easily estimated with multiplying the first three eigenvectors, 3PC of the source`s $\mathrm{S}_{\mathrm{FCS}}$, by the specifically weighted coefficients of the target.

$$
\text { Estimated } \hat{S}_{F C S}=\alpha_{\text {target }} \times 3 P c_{\text {source }}
$$

The last step is continued by changing the estimated $\hat{\mathrm{S}}_{\mathrm{FCS}}$ to estimated $\hat{\mathrm{S}}$ for each pixels of the target in aid of $\mathrm{L}$ and finalized the method by recombining $\hat{\mathrm{S}}$ with its corresponding $\mathrm{g}$ and $\mathrm{k}$. This gives new values converted target, $\breve{I}$ for each pixels of the target image. Figure 4 shows flowchart of the proposed color converting method using dichromatic model, decomposition theory and principal component analysis. 


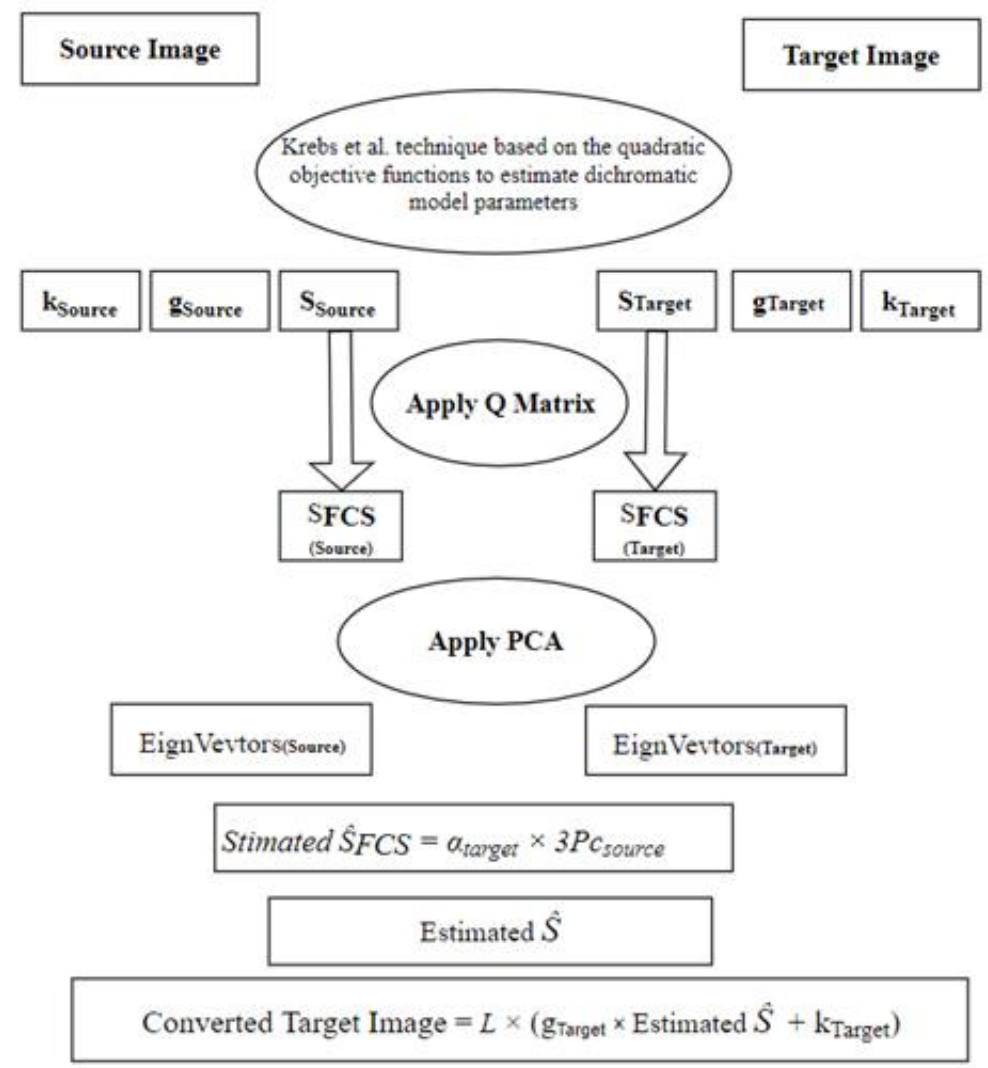

Figure 4. Flow chart of the proposed color converting using decomposition theory and principal component analysis.

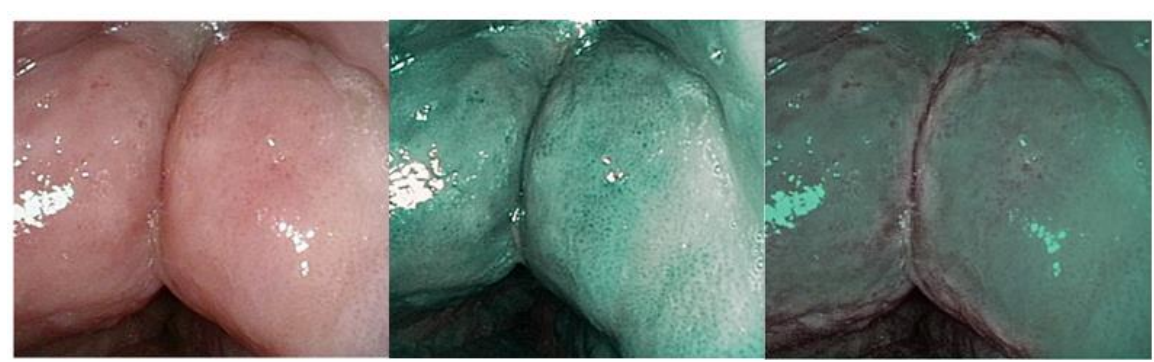

Figure 5. The target image(left), the source image(middle), the converted target image(right).

Figure 5 shows the result of the converted color space of the source image to the new target image in comparison with the original target image. It can be seen that the suggested method can provide the possibility to convert the color spaces between images. For more clarification, the color gamut of the source, target, and converted target images are shown in Figure 6. As indicated, the color gamut of the converted target image gets closer to the NBI image color space after applying the suggested color converting technique. 


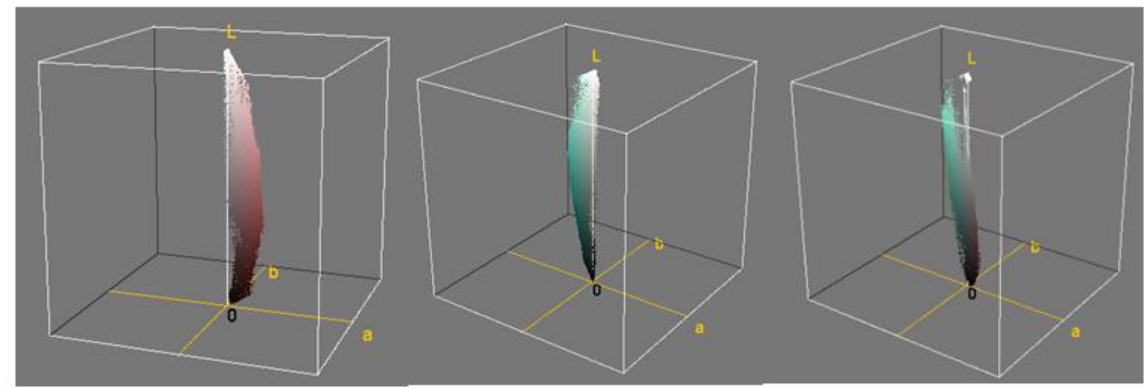

Figure 6. The CIELAB color gamuts of : the target image(left), the source image(middel) and the converted target image(right).

\section{CONCLUSION}

A hands-on novel technique was introduced to convert the color gamut atmosphere of a narrowband image into the conventional target image. This new combined technique would be valuable in the field of medical endoscopic color image enhancement and clinical diagnoses.

Firstly, the quadratic objective functions for the dichromatic model by Krebs et al. applied to estimate optical parameters such as intrinsic image, shading, and specularity from the source image (narrowband endoscopic color image) and the target image (conventional endoscopic color image).

Secondly, the decomposition theory was introduced by Cohen and Kappauf as an orthogonal projector to the Wyszecki`s spectral decomposition theory, employed to obtain the fundamental stimuli of the intrinsic image belonging to the target and the source images.

Finally, by using the principal component analysis classic technique, the first three eigenvectors of the fundamental stimuli of the target image were taken and replaced by eigenvectors of the fundamental stimuli of the source image.

The resulting image and the color gamut's were showed that this new algorithm could be feasibly applied for color space converting between color images, such as Narrow Band Images(NBI), into the conventional endoscopic color images.

\section{ACKNOWLEDGMENT}

This study was supported by the French Research National Agency (ANR) program EMMIE under the grant agreement 15-CE17-0015.

\section{REFERENCES}

[1] S. Tanaka, S. oka, M. hirata, S. Yoshida, I. Kaneko and K. Chayama, (2006) "Pit pattern diagnosis for colorectal neoplasia using narrow band imaging magnification," Digestive Endoscopy 18(Suppl. 1), pp. S52-S56.

[2] P. Lukes et al. (2013) "Narrow Band Imaging (NBI)", Endoscopy, IntechOpen, Edited by S. Amornyotin, Chapter 5.

[3] R. Saito and H. Kotera, (2005)"Gamut mapping adapted to image contents," Proc. Congress of the International Colour Association (AIC 05), Granada, Spain, pp. 661-664. 
[4] X. Xiao, L. Ma, (2006) "Color transfer in correlated color space," In VRCIA '06: Proc. of the 2006 ACM international conference on virtual reality continuum and its applications, pp. 305309.

[5] E. Reinhard, M. Ashikhmin, B. Gooch, and P. Shirley, (2001) "Color transfer between images," IEEE Comput.Graphics Appl., pp. 34-41.

[6] H. Kotera, Y. Matsusaki, T. Horiuchi, R.Saito, "Automatic color interchange between images," Proc. Congress of the International Color Association (AIC 05), Granada, Spain, pp. 1019 -1022.

[7] R. Saito, T. Horiuchi, and H. Kotera, (2005)“Scene color interchange using histogram rescaling," Proc. IS\&T's International Conference on Digital Printing Technologies (NIP22), Denver, Colorado, pp. 378-381, 2006.

[8] R. Saito, H. Okuda, T. Horiuchi and S. Tominaga, (2007) "Scene-to-scene color transfer model based on histogram rescaling," Proc. Midterm Meeting of the International Color Association (AIC 07), Hangzhou, China, pp. 122-125.

[9] S. Gorji Kandi, K. Ansari, (2011)“ Transforming color space between images using rosenfeldkak histogram matching technique,” 4th International Color and Coatings Congress (ICCC 2011), Tehran, Iran.

[10] E. Reinhard, M. Ashikhmin, B. Gooch, and P. Shirley, (2001)“Color transfer between images,” IEEE Comput. Graphics, pp.34-41.

[11] Y. Chang, S.Saito, and M. Nakajima, (2007)"Example-based color transformation of image and video using basic color categories," IEEE Trans. Image Process, vol. 16,no. 2, pp. 329-336.

[12] S. Paris, S. W. Hasinoff, and J. Kautz, (2011)“Local Laplacian filters: Edge-aware image processing with a Laplacian pyramid," ACM Trans. Graph., vol. 30, no. 4, pp. 1-12.

[13] A. Abadpour, S. Kasaei, (2007)“An efficient PCA-based color transfer method,” J. Visual Communication and Image Representation.

[14] A. Dhanve1, G. Chhajed, (2014)“Review on color transfer between images," International Journal of Engineering Research and General Science Vol. 2, Issue 6, Oct. -Nov.

[15] A. Krebs, Y. Benezeth, F. Marzani, (2017)“Quadratic objective functions for dichromatic model parameters estimation," in: IEEE International Conference on Digital Image Computing: Techniques and Applications (DICTA).

[16] S. A. Shafer, (1985)"Using color to separate reflection components," Color Research \& Application 10 (4), pp.210-218.

[17] J. B. Cohen and W. E. Kappauf, (1982)“Metameric color stimuli, fundamental metamers, and Wyszecki's metameric blacks,” Am. J. Psychol. 95, pp. 537-564.

[18] F. Viénot, H. Brettel, (2014)"Visual properties of metameric blacks beyond cone vision," Journal of the Optical Society of America, Vol. 31,Issue 4, pp. A38-A46.

[19] Y. Mohamed, Y. Abdallah and T.Alqahtani,(2019) "Research in Medical Imaging Using Image Processing Techniques,”Medical Imaging - Principles and Applications, IntechOpen, Edited by Y. Zhou, Chapter 5.

[20] B.Selvapriya and B. Raghu,(2018) “A Color Map for Pseudo Color Processing of Medical Images,” International Journal of Engineering \& Technology, 7 (3.34) 954-958. 
[21] K. Ansari, S. Moradian, and S.H. Amirshahi,(2005)" Ideal Compression of Reflectance Curves by the use of Fundamental Color Stimuli", 10th Congress of the International Colour Association, AIC Colour 2005, Granada, Spain.

\section{AUTHORS}

Keivan Ansari received his Ph.D. in color engineering from Amirkabir University (Polytechnic of Tehran),2005. He is an assistant professor in Color Imaging \&Color Image Processing research group in Color for Science \& Technology Institute, Tehran, IRAN. He is currently pursuing his postdoctoral at the ImViA laboratory in the Bourgandy university, Dijon, FRANCE. His work has focused on the development of Color Physics and its application in image processing.

Alexandre Krebs received the B.S., M.S. and Ph.D. in computer sciences and image instrumentation from the University of Burgundy (France) in 2019. He is currently a temporary lecturer and research assistant in the engineering school ESIREM in Dijon, France. His research includes digestive endoscopy, Narrow Band Imaging, multispectral imaging, stomach lesions, machine Learning, transfer learning, Inverse problem, and optimization.

Yannick Benezeth is associate professor at the Univ. Bourgogne Franche-Comté (France). He obtained his Ph.D. in computer science from the Univ. of Orléans in 2009. He also received the engineering degree from the ENSI de Bourges and the MS degree from the University of Versailles-Saint-Quentin-en-Yvelines in 2006. His research interests include biomedical engineering, image processing, and video analytics. Application areas include video health monitoring and endoscopy.
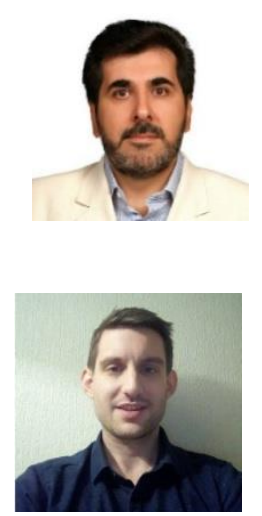

Franck Marzani received his M.Sc. in computer science from the University of Rennes, France in 1989. He obtained his Ph.D. in computer vision and image processing from the University of Burgundy, Dijon, France in 1998. He received his "Habilitation à Diriger les Recherches" in 2007 and he is a full professor since 2009. he is currently the head of the ImViA research laboratory (Imaging \& Computer Vision) at the University of Burgundy. His research interests include

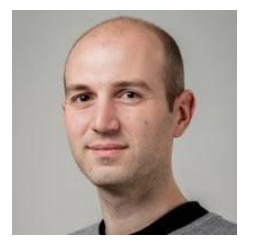
acquisition and analysis of images.

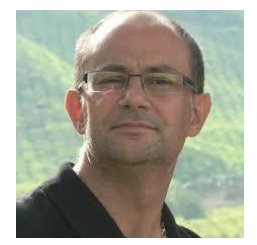

